\title{
Solitary Fibrous Tumor - Less Common Neoplasms of the Pleural Cavity
}

\author{
Sarka Vejvodova, MD, ${ }^{1}$ Vladimir Spidlen, MD,${ }^{1}$ Petr Mukensnabl, MD, PhD,${ }^{2}$ \\ Gabriela Krakorova, MD, PhD, ${ }^{3}$ Jiri Molacek, MD, PhD, ${ }^{1}$ \\ and Josef Vodicka, $\mathrm{MD}, \mathrm{PhD}^{1}$
}

\begin{abstract}
Purpose: solitary fibrous tumors (SFT) represent a heterogeneous group of primary pleural neoplasms with a low incidence rate and of which the biological origin, which consists of mesenchymal cells, is uncertain.

Methods: The authors present herewith a retrospective analysis of 22 patients with SFTs who were diagnosed and surgically treated between the years 2000-2015. The preoperative tumors were successfully verified morphologically by transthoracic core needle biopsy under CT control in $27.3 \%$ of patients. Surgical approaches were either posterolateral thoracotomy or videothoracoscopy. The follow-up median was 45 months (range 1-188 months). Results: Twenty tumors were surgically removed radically, two tumors were found to be unresectable due to the considerable tumor size. From histological point of view $81.8 \%$ of tumors were SFT with low malignant potential, $18.2 \%$ of tumors with high malignant potential. Despite the radical extirpation of the SFT, it relapsed in two patients.

Conclusion: The gold standard of SFT treatment is radical surgical removal; however, patients at risk of recurrence require additional follow-ups. The results of adjuvant therapy in recurrent and malignant forms of SFTs are the subject of discussion and further study.
\end{abstract}

Keywords: solitary fibrous tumor, pleura, De Perrot classification, diagnosis, surgical treatment

\section{Introduction}

The solitary tumor of the pleural cavity was described for the first time by Wagner in $1870 .{ }^{1)}$ During the following

${ }^{1}$ Clinic of Surgery, Faculty of Medicine in Pilsen, Charles University in Prague, University Hospital Pilsen, Pilsen, Czech Republic ${ }^{2}$ Sikl Department of Pathology, Faculty of Medicine in Pilsen, Charles University in Prague, University Hospital Pilsen, Pilsen, Czech Republic

${ }^{3}$ Clinic of Pneumology and Phtiseology, Faculty of Medicine in Pilsen, Charles University in Prague, University Hospital Pilsen, Pilsen, Czech Republic

Received: April 26, 2016; Accepted: July 25, 2016

Corresponding author: Josef Vodicka, MD, PhD. Clinic of Surgery, Faculty of Medicine in Pilsen, Charles University in Prague, University Hospital Pilsen, Avenue Svobody 80, 30460 Pilsen, Czech Republic

Email: vodicka@fnplzen.cz

(C)2017 The Editorial Committee of Annals of Thoracic and Cardiovascular Surgery. All rights reserved. years only isolated cases of similar findings were mentioned which were due to their intimate relationship to pleural tumors based on the mesothelial layer. In 1931, Klemperer and Rabin classified these tumors as (so called) benign (localized) mesotheliomas with no proven asbestos exposure in contrast to malignant (diffuse) mesothelioma, for which such a correlation was proven. ${ }^{1,2)}$ It was in the $1980 \mathrm{~s}$, on the basis of these immunohistochemical methods, that the origin of these solitary tumors was determined to be based not on the pleural mesothelium lining but on the mesenchymal cells layer, deposited subpleurally. ${ }^{3)}$ This finding led to changes to the originally inconsistent terminology for these tumors. Tumors formerly identified as so-called benign mesotheliomas, benign pleural fibromas, submesotelial fibromas, pleural fibromyxomas, etc., were newly classified as so-called solitary fibrous tumors (SFT). ${ }^{4,5)}$ These are now defined as primary pleural neoplasms originating from the mesenchymal tissue which 
produces vimentin and CD34 (a transmembrane glycoprotein expressed by mesenchymal stem cells), or which gives a positive reaction to STAT6 (protein transmitting signals from the receptor complex into the nucleus and gene expression activator). ${ }^{1,6}$

\section{Material and Methods}

This is a retrospective analysis of 22 patients with solitary fibrous pleural tumors that were diagnosed and surgically operated by the authors between the years 2000-2015. The group of operated patients included 16 women and six men whose mean age was 59.6 years, with an age range of 28-73 years. The laterality of the SFT occurrence was almost balanced, 10 tumors were located on the left side, 12 on the right. The diagnosis of SFT was based on standard investigation methods for surgical diseases of the lung and pleura i.e. computer tomography (CT), magnetic resonance, fibrobronchoscopy, etc. The successful preoperative verification of the tumors was based on morphology determined through a transthoracic core needle biopsy under CT control in $27.3 \%$ of patients. The surgical approaches were either posterolateral thoracotomy or videothoracoscopy. Histopathological examinations were performed on the removed tumors in a standard manner using the haematoxylin-eosin dying method, and by immunohistochemistry. SFT were classified according to the number of mitosis in $2 \mathrm{~mm}^{2}$ (World Health Organization classification of lung and pleural tumors from 2015) on two categories - tumors with low malignant potential ( $\leq 4$ mitosis $/ 2 \mathrm{~mm}^{2}$ ) and tumors with high malignant potential ( $>4$ mitosis $/ 2 \mathrm{~mm}^{2}$ ). The median of the followup group was 45 months (range 1-188 months).

\section{Results}

An accidental finding in a chest X-ray for other indications appeared in 10 asymptomatic patients. Another 12 patients were screened for pre-existing problems which mostly included increasing dyspnoea $(91.5 \%$ of the clinical manifestations), followed by haemoptysis and weight loss. Paraneoplastic Pierre-Marie-Bamberg syndrome (pain, swelling and redness in the area of the small leg joints and subfebrile temperature) was found in one patient.

Twenty tumors were surgically removed radically, three via videothoracoscopy, the remaining cases via thoracotomy. In two cases the tumors were found to be

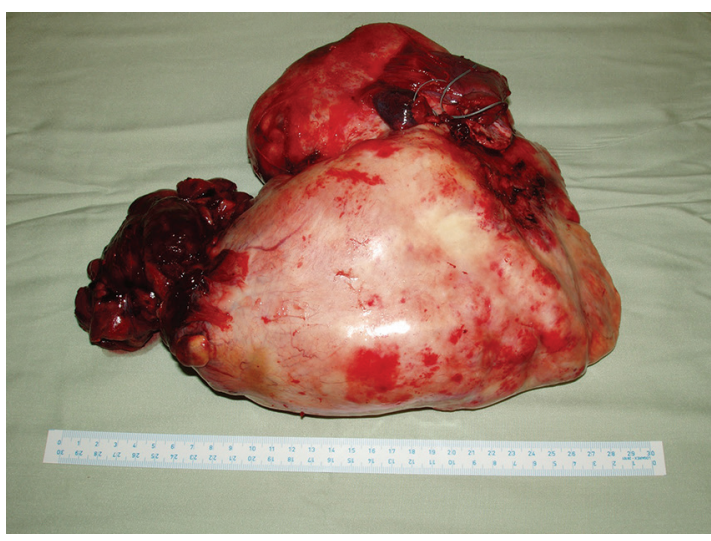

Fig. 1 Solitary fibrous tumor removed from the left pleural cavity (max. size $30 \mathrm{~cm}$, weight $2500 \mathrm{~g}$ ).

unresectable due to the considerable size of the tumors and invasion into mediastinum and magistral vessels. In 11 patients the SFTs originated on the visceral pleura, in seven patients in the parietal pleura, in one patient from both layers of pleura, whilst deposition in the lung parenchyma was found in three cases (upper right twice, right lower lung lobe once). These intraparenchymatous findings were treated by wedge resection in two cases and once by a lobectomy of the respective lobe. This also had to be performed in one case of bulky intrapleurally localized tumor that lead to the irreversible loss of function of the entire lung lobe due to its oppression. In a rare case of the occurrence, four discrete bearings in one pleural cavity were found, two on the visceral pleura and two on the parietal pleura. The tumor sizes ranged from $3-30 \mathrm{~cm}$ lengthwise, with the greatest recorded weight being $2500 \mathrm{~g}$ (Fig. 1). From histological point of view $81.8 \%$ of tumors were SFT with low malignant potential, $18.2 \%$ of tumors with high malignant potential. Patients' data are stated in the Table 1.

In the group there were no significant peri- or postoperative complications. Patients were hospitalized on average for 7 days. One patient with high malignant potential SFT subsequently received adjuvant chemotherapy with etoposide-carboplatin, the other patients were followed up. SFT relapse occurred in two patients despite the original radical extirpation. The first case was in a 68-year-old woman with intraparenchymatous low malignant potential SFT in the upper lobe of the right lung that was removed by a non-anatomical resection. A relapse occurred four years after the initial surgery and was successfully resolved through a right-upper lobectomy. The second case of relapse was in a 69-year-old 


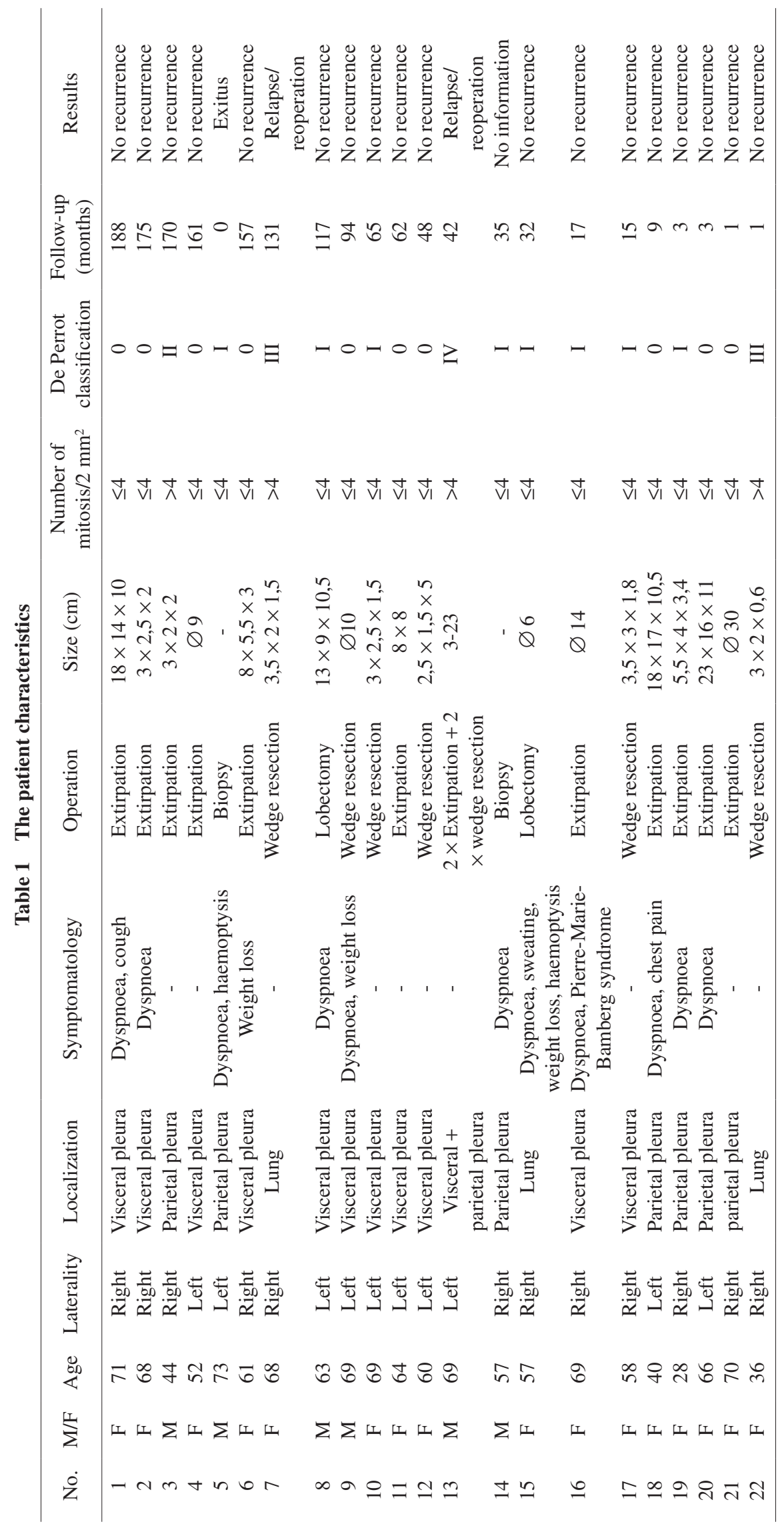




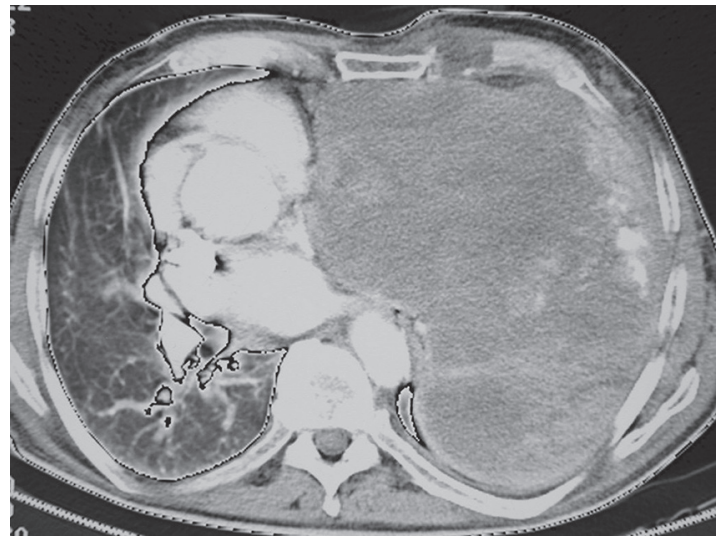

Fig. 2 CT image of an inoperable solitary fibrous tumor in the left pleural cavity (axial section). CT: computer tomography

man which occurred 18 months after the removal of multiple SFT foci from the right pleural cavity. Radical extirpation of the recurrent bearings was successfully performed. One patient suffering from inoperable SFT died on the fourth postoperative day due to heart failure caused by oppression by the huge tumor (Fig. 2).

\section{Discussion}

SFTs makes up less than $5 \%$ of all primary pleural tumors. The incidence rate is 2.8 cases per 100000 population..$^{2)}$ So far, 900 cases of this disease have been described in literature. ${ }^{7,8)}$ Since SFTs are derived from mesenchymal cells, it is also possible that they originate extrapleurally and are therefore better known as extrapleural solitary fibrous tumors (ESFT). They can be found for example in the nasal cavities, orbits, mouth, salivary glands, thyroid, kidney, spinal cord and elsewhere. ${ }^{5)}$ In rare cases, SFTs also occur in the lung parenchyma where they originate from the mesenchymal cells transformation in interlobular septa. ${ }^{1)}$ According to Sakurai et al., it has been published only 11 cases of intrapulmonary localized SFT until 2008.9) Compared with these data we can consider our presentation of three cases (in a group of 22 patients) as a relatively rare. Pleural SFTs usually occur singly, however cases of multiple occurrence in a pleural cavity have been reported too. ${ }^{10)}$ The peak incidence rate is in those people in their $60 \mathrm{~s}$ and 70 s, whereby the proportion of men to women is approximately equal. $1,5,11,12$ )

Solitary fibrous tumors usually represent themselves as an accidental finding as a result of an imaging examination

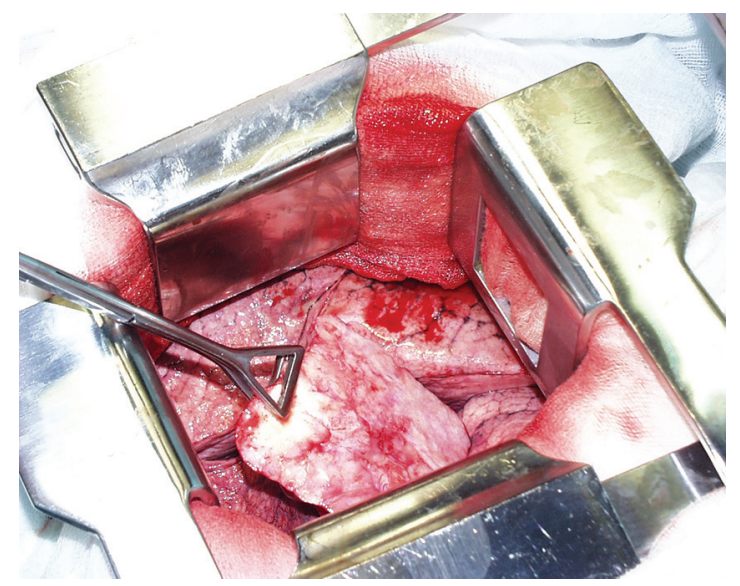

Fig. 3 Solitary fibrous tumor of the right pleural cavity shanked to the pleura of the interlobar fissure (photo obtained during surgery).

for other reasons. Nevertheless, up to half of the cases present themselves through their clinical manifestation. ${ }^{7,13-15)}$ The symptoms may either be intrathoracic due to the suppression of the lung as a result of tumor growth, or by formed pleural effusion, as well as extrathoracic due to the production of specific substances by cancer cells proper.") The most common intrathoracic symptoms are coughing, chest pain, dyspnoea and haemoptysis. ${ }^{17,14)}$ The presence of pleural effusion was reported in up to $16 \%$ of cases, especially in high malignant potential SFT. ${ }^{5,8)}$ Weight loss was described in $10 \%$ of patients. ${ }^{1,4)}$ Extrathoracic (paraneoplastic) symptoms may include joint pain and finger paraesthesia caused by the production of liver-derived growth factor (hepatocyte growth factor - HGF) or the excessive production of hyaluronic acid as part of Pierre-MarieBamberg syndrome (hypertrophic pulmonary osteoarthropathy) or intermittent hypoglycaemia (Doeg-Potter syndrome) caused by the production of IGF-II (insulin-like growth factor II). ${ }^{16)}$ The Doeg-Potter syndrome ranges from $2 \%$ to $4 \%$, rarely up to $14 \%$, and is described more frequently in tumors localized in the right pleural cavity with a size more than $20 \mathrm{~cm}$ and with high tumor cell mitotic potential. ${ }^{7,817)}$ The manifestation of Pierre-Marie-Bamberg syndrome is reported to be 35\%. ${ }^{11)}$ In rare cases, gynecomastia and galactorrhoea have also been reported in some patients. ${ }^{1)}$

Most solitary fibrous tumors are detected early during examinations using imaging methods. On chest $\mathrm{X}$-rays, the tumors usually appear as a solitary, well-circumscribed homogeneous expansion, sometimes associated with pleural effusion, most commonly in the middle and lower parts of the pleural cavity. ${ }^{1)}$ Computer tomography examinations 
Table 2 De Perrot classification of the solitary fibrous tumor of the pleura

\begin{tabular}{ccc}
\hline Stadium & Tumor morphology & Recurrence prognosis \\
\hline 0 & Stem SFT with no signs of malignancy & $2 \%$ \\
I & Adjacent SFT with no signs of malignancy & $8 \%$ \\
II & Stem SFT with histological evidence of malignancy & $14 \%$ \\
III & Adjacent SFT with histological evidence of malignancy & $63 \%$ \\
IV & Multiple synchronous metastatic tumors & \\
\hline
\end{tabular}

SFT: solitary fibrous tumor

usually show a low density lesion (25-40 Hounsfield units) with a rich vascular supply. Lung oppression with atelectasis, the presence of central necrosis, or an inhomogeneous image of the tumor, can make it difficult to differentiate SFTs from other pleural neoplasms and lung cancers, or to differentiate SFT with low malignant potential. ${ }^{2,4)}$ CT angiography and magnetic resonance help to make the diagnosis more accurate due to the more detailed information they provide with regards to the fibrous component of the expansion and its relation to the surrounding structures. ${ }^{18,19)}$ The successful morphological verification of the tumor was carried out by transthoracic core needle biopsy under CT control. The success rate is $38 \%-50 \%$ (27.3\% in our sample). ${ }^{1,2,4,7,8,12)}$

SFTs are usually well-circumscribed having an ovoid or spherical shape, covered by a fibrous sheath of a whitish color, under which the rich vascular supply shines through. The sometimes also consist of multiple nodes. When cut, they are whitish-grey, with noticeable foci of necrosis and hemorrhage. ${ }^{1)}$ Histologically, SFTs are formed by uniform spindle fibroblasts with varying degrees of cellularity in the different parts of the tumor. Deposits of stromal hyalinization are frequent too, which are mostly situated around blood vessels. Foci with a fascicular or storiform arrangement can also be found on occasions. Immunohistochemistry examinations of the tumor cells $\mathrm{CD} 34, \mathrm{Bcl} 2$ and $\mathrm{CD} 99$ are usually positive, but the positivity is not entirely specific. ${ }^{15)}$ A positive STAT6 reaction is therefore considered to be the most evident ( $>95 \%)$. Furthermore, certain tumors may also be smooth muscle actin (SMA), epithelial membrane antigen (EMA), desmin, S100 protein or keratin positive. ${ }^{20)}$ The size of SFTs range from a few millimeter to the tens of centimeter (0.8-26 $\mathrm{cm}) .{ }^{21)} \mathrm{In}$ up to $80 \%$ of cases they originate on the visceral pleura and are connected to it by a shank or wide anneal, typically in the interlobar fissure areas in the middle or bottom parts of the pleural cavity (Fig. 3). 12,14,21)

From the point of view of the biological behavior, the majority of solitary fibrous tumors are with low malignant potential. Nevertheless, in literature there are also descriptions of the incidence of variants with high malignant potential in the range of $7 \%-60 \%, 1,2,4,12,15)$ The mitotic activity of the tumor is not high, usually less than 5 mitoses per $2 \mathrm{~mm}^{2}$. A higher number of mitoses and hypercellularity is usually the sign of the aggressive behavior of the tumor. Potential criteria for malignant SFTs have not yet been precisely specified and unified. According to some authors, tumors of a larger size $(>8-13 \mathrm{~cm})$ infiltrating the surrounding area, with an increased cellularity, higher number of central necrosis and hemorrhage, or associated with effusion, have a higher tendency to show malignant behavior. , $7,12,22$ ) $^{2}$ However, other studies refer to the fact that the size of the tumor, its location, the presence of symptoms, or the fact that it is based on the visceral or parietal pleura, are not unambiguous evidence of the increased possibility of tumor recurrence and shortened survival interval. ${ }^{8,15)}$ The expression of the p16 SFT protein is not significantly associated with its recurrence. ${ }^{15)}$ High malignant potential SFTs frequently metastasize to the lungs, bones, liver, kidney, adrenal glands and spleen, and in rare cases to the brain. ${ }^{1,23)}$ According to the macroscopic findings during surgery and histological analysis, De Perrot proposed an SFT classification on the basis of which it is possible to accurately predict their future behavior (Table 2). . $^{\text {) }}$

The gold standard of SFT treatment is their radical surgical extirpation via either a mini-invasive approach or a thoracotomy. The limit for a mini-invasive procedure is determined by the size of the tumor. The recommended SFT size is $5 \mathrm{~cm}$ in diameter. ${ }^{1,2,4,12)} \mathrm{A}$ fundamental prerequisite for successful treatment is an R0 resection, including adhesions between it and the surrounding pleura. This is because they can also be a source of disease recurrence. ${ }^{15,21)}$ When SFT infiltration into the lung parenchyma appears, its non-anatomical or anatomical resections are indicated depending on the extent of the affection. Radical surgery is considered for low 
malignant potential SFT, however, patients must continue to be carefully monitored for the risk of recurrence despite the original radical removal of the tumor. ${ }^{15,24)}$ The administration and success of adjuvant therapy (chemotherapy, radiotherapy) for recurrences and SFT with high malignant potential are, due to the relatively small number of patients, still subject to discussion. ${ }^{1,2,6,25)}$ The risk of SFT recurrence can be up to $30 \%$ (in our sample $9,1 \%$ ), with five-year survival rates after radical SFT removal of around $90 \%$, and a ten-year survival rate of around $86 \% .5,22)$

\section{Conclusion}

Solitary fibrous tumors represent themselves as a heterogeneous group of primary pleural neoplasms of an uncertain biological nature originating from mesenchymal cells. Malignant behavior can be expected in tumors with concurrent paraneoplastic symptoms, pleural effusion, larger size, infiltration into surrounding tissues, with increased cellularity and a plurality of central necrosis and hemorrhage. At present, accurate information on the biological nature and the risk of SFT recurrence is given under the De Perrot classification. However, as shown by our experience, tumors of a larger size, with pleural effusion formation and paraneoplastic symptoms, can be quite benign and treatable through radical surgery with high long-term survival rates without relapse or disease process generalization. The gold standard of SFT treatment is their radical surgical removal. The administration and results of adjuvant therapy in recurrent and SFTs with high malignant potential are the subject of discussion and further studies.

\section{Disclosure Statement}

The authors declare that no conflicts of interest exist.

\section{References}

1) Abu Arab W. Solitary fibrous tumours of the pleura. Eur J Cardiothorac Surg 2012; 41: 587-97.

2) Liu J, Cai C, Wang D, et al. Video-assisted thoracoscopic surgery (VATS) for patients with solitary fibrous tumors of the pleura. J Thorac Oncol 2010; 5: 240-3.

3) El-Naggar AK, Ro JY, Ayala AG, et al. Localized fibrous tumor of the serosal cavities. Immunohistochemical, electron-microscopic, and flow-cytometric DNA study. Am J Clin Pathol 1989; 92: 561-5.
4) Magdeleinat P, Alifano M, Petino A, et al. Solitary fibrous tumors of the pleura: clinical characteristics, surgical treatment and outcome. Eur J Cardiothorac Surg 2002; 21: 1087-93.

5) Szkorupa M, Klein J, Bohanes T, et al. [Solitary fibrous tumor of pleural cavity]. Rozhl Chir 2010; 89: 750-3. (in Czech)

6) Leng XF, Xian L, Qin JJ, et al. Malignant solitary fibrous tumor of pleura accompanied with first symptoms of chest pain and hemoptysis: a case report. Ann Thorac Cardiovasc Surg 2012; 18: 251-5.

7) Rena O, Filosso PL, Papalia E, et al. Solitary fibrous tumour of the pleura: surgical treatment. Eur J Cardiothorac Surg 2001; 19: 185-9.

8) Lococo F, Cesario A, Cardillo G, et al. Malignant solitary fibrous tumors of the pleura: retrospective review of a multicenter series. J Thorac Oncol 2012; 7: 1698-706.

9) Sakurai H, Tanaka W, Kaji M, et al. Intrapulmonary localized fibrous tumor of the lung: a very unusual presentation. Ann Thorac Surg 2008; 86: 1360-2.

10) Tastepe I, Alper A, Ozaydin HE, et al. A case of multiple synchronous localized fibrous tumor of the pleura. Eur J Cardiothorac Surg 2000; 18: 491-4.

11) Dynes MC, White EM, Fry WA, et al. Imaging manifestations of pleural tumors. Radiographics 1992; 12: 1191-201.

12) Thakkar RG, Shah S, Dumbre A, et al. Giant solitary fibrous tumour of pleura -an uncommon intrathoracic entity- a case report and review of the literature. Ann Thorac Cardiovasc Surg 2011; 17: 400-3.

13) Abe M, Nomori H, Fukazawa M, et al. Giant solitary fibrous tumor of the pleura causing respiratory insufficiency: report of 3 cases. Ann Thorac Cardiovasc Surg 2014; 20: 441-4.

14) Enon S, Kilic D, Yuksel C, et al. Benign localized fibrous tumor of the pleura: report of 25 new cases. Thorac Cardiovasc Surg 2012; 60: 468-73.

15) Liu CC, Wang HW, Li FY, et al. Solitary fibrous tumors of the pleura: clinicopathological characteristics, immunohistochemical profiles, and surgical outcomes with long-term follow-up. Thorac Cardiovasc Surg 2008; 56: 291-7.

16) Maeda S, Sugita M, Sagawa M, et al. Solitary fibrous tumor of the pleura suddenly induced hypoglycemia before surgical treatment. Ann Thorac Cardiovasc Surg 2011; 17: 293-6.

17) Li Z, Wang J, Zhu Q, et al. Huge solitary fibrous tumor of the pleura with hypoglycemia and hypokalemia: a case report. Ann Thorac Cardiovasc Surg 2014; 20: 165-8.

18) Usami N, Iwano S, Yokoi K. Solitary fibrous tumor of the pleura: evaluation of the origin with $3 \mathrm{D} \mathrm{CT}$ angiography. J Thorac Oncol 2007; 2: 1124-5.

19) Yao $H, H u Z$, Shi $H$, et al. Intrathoracic solitary fibrous tumor: a report of 3 cases and review of the literature. Chinese-German J Clin Oncol 2012; 11: 660-4. 
20) Goodlad JR, Fletcher CD. Solitary fibrous tumour arising at unusual sites: analysis of a series. Histopathology 1991; 19: 515-22.

21) Orki A, Kosar A, Akin O, et al. Solitary fibrous tumor of the pleura. Thorac Cardiovasc Surg 2008; 56: 287-90.

22) Boddaert G, Guiraudet P, Grand B, et al. Solitary fibrous tumors of the pleura: a poorly defined malignancy profile. Ann Thorac Surg 2015; 99: 1025-31.
23) Ozeki N, Kawaguchi K, Taniguchi T, et al. Primary pulmonary solitary fibrous tumour with brain metastases. Eur J Cardiothorac Surg 2014; 45: 386-8.

24) Cardillo G, Facciolo F, Cavazzana AO, et al. Localized (solitary) fibrous tumors of the pleura: an analysis of 55 patients. Ann Thorac Surg 2000; 70: 1808-12.

25) Bylicki O, Rouvière D, Cassier $\mathrm{P}$, et al. Assessing the multimodal management of advanced solitary fibrous tumors of the pleura in a routine practice setting. J Thorac Oncol 2015; 10: 309-15. 